\title{
Fast Slab Gel Electrophoretic Separation of DNA Fragments with a Short Migration Distance Using Thermal Lens Microscope
}

\author{
Jinjian ZhenG*, Tamao OdAKE**, Takehiko KITAMORI*,** and Tsuguo SAWADA*广 \\ *Department of Applied Chemistry, School of Engineering, The University of Tokyo, \\ Hongo, Bunkyo, Tokyo 113-8656, Japan \\ **Kanagawa Academy of Science and Technology, 3-2-1 Sakado, Takatsu, Kanagawa 213-0012, Japan
}

\begin{abstract}
We demonstrated fast separation of DNA fragments in a short slab gel through the minimization of band-broadening. A discontinuous buffer system was used to reduce band-broadening from sample introduction, and a high spatial resolution thermal lens microscope (TLM) was used to reduce such an effect from the detector. Bands of DNA fragments were sharpened into $100 \mu \mathrm{m}$, which was about $1 / 10$ of the bandwidths in conventional continuous slab gel electrophoresis. The 100 base pair ladder DNA sample, which ranges from $100 \mathrm{bp}$ to $2000 \mathrm{bp}$, was completely separated within 15 min with a migration distance of $18 \mathrm{~mm}$ and was detected by TLM after silver staining. These results suggested the possibility of fast separation of DNA fragments in a miniaturized discontinuous slab gel. As well as the inherent properties of parallel separation of slab gel, high throughput separation can be obtained, which is significant for DNA analyses such as DNA sequencing, genetic diagnosis and forensic identification analyses.
\end{abstract}

Keywords Electrophoresis, slab gel, DNA, miniaturization, thermal lens microscope, discontinuous buffer

Many important biological techniques, such as DNA sequencing ${ }^{1,2}$, restriction mapping ${ }^{3,4}$, southern blotting 5 and Dnase footprinting 6 , rely on the use of extremely specific enzymes, in combination with an electrophoretic separation step to provide the desired information. Most of these electrophoresis separations are carried out in slab gels which are typically $20-100 \mathrm{~cm}$ in length and $200-2000 \mu \mathrm{m}$ in thickness, and then followed by radioisotope, UV-visible absorbance or laser induced fluorescence (LIF) detection. Usually it takes several hours to more than $10 \mathrm{~h}$ to run one separation.

In practice, however, it is usually necessary to treat an enormous number of DNA samples as fast as possible. Therefore, high throughput analysis is desired. The throughput of an electrophoresis system can be improved either by (1) shortening the separation time or by (2) performing the separations in parallel.

To obtain fast separation, researchers have developed ultrathin slab gel electrophoresis ${ }^{7-9}$, capillary electrophoresis $^{10-12}$ and microchip electrophoresis. ${ }^{13,14}$ The fundamental advantage of these techniques lies in the increased Joule heat dissipation efficiency resulting from the increased surface area-to-volume ratio of the gel. Hence a higher field can be applied and thus the separation time is shortened. It has been reported that ten times faster separation can be obtained using these techniques. ${ }^{8}$ Ultrathin slab gel electrophoresis also allows many samples to be run in parallel and allows comparison of the electrophoresis patterns of related

† To whom correspondence should be addressed. samples side by side, which is often necessary for such techniques as DNA sequencing, Dnase footprinting and the study of DNA structural polymorphism ${ }^{7}$. However, ultrathin slab gel electrophoresis usually encounters a difficulty in sample introduction and gel casting. Although it has been reported that parallel separations can also be performed in capillary electrophoresis or microchip electrophoresis, these two technologies are limited by a difficulty in system arrangement and difference of separation performance which is attributed to the different surface conditions between columns. Therefore, these techniques are still not used in routine DNA analyses.

Here we propose another technique for fast separation, which is based on the idea that, if the band-broadening can be minimized, it is possible to finish the separation in a short slab gel within a short separation time. Considering the inherent properties of parallel separations in slab gel, we thought that it would be possible to obtain high throughput separation.

In this research, we obtained fast separation in a slab gel with a short migration distance. We used a discontinuous buffer system to minimize band-broadening from sample introduction. After silver staining, the separated bands were detected by a high spatial resolution detector, a thermal lens microscope (TLM) to reduce band-broadening from the detector. The 100 base pair ladder DNA was separated in 15 min with a migration distance of $18 \mathrm{~mm}$. Both the separation time and the migration distance were about one-tenth of those in a conventional continuous slab gel. 


\section{Experimental}

\section{Instrumentation}

A schematic illustration of the laboratory-constructed TLM is shown in Fig. 1. The emission line of an aircooled argon ion laser $(488 \mathrm{~nm}, 4 \mathrm{~mW})$ was used as the excitation beam after mechanical chopping. The modulated beam was transmitted through a dichroic mirror, adjusted coaxially with the probe beam (633 nm, 1 $\mathrm{mW}$ ) which came from a He-Ne laser and was reflected off the dichroic mirror. Both beams were introduced into an optical microscope and focused onto the surface of a gel using a $40 \times \mathrm{NA} 0.65$ objective lens. The excitation beam energy absorbed by a sample was changed into heat which made the temperature of the focused area distribute Gaussianly around the excitation beam axis. That is, the temperature at the center of the light spot was higher than that on the periphery. The difference in temperature caused a difference of refractive index. In the case of polyacrylamide gel, the refractive index had a negative correlation with temperature, which created something like a concave lens in the gel. That is called the photothermal lens effect. Therefore, divergence of the probe beam changed when it transmitted through the photothermal lens and the amount of probe light entering the pinhole and being collected by the photodiode changed. The excitation beam was cut by a filter so that only probe light was detected. The changes in the probe beam indicated the degree of the photothermal lens. After silver staining, the slab gel was fixed onto a 3-dimensional scanning stage with a precision of $1 \mu \mathrm{m}$. TLM scanning of the separated DNA pattern was done by driving the stage using a personal computer.

All separations were performed in a mini-electrophoresis apparatus provided by Nihoneido (Tokyo, Japan).

\section{Chemicals}

The 100 base pair ladder DNA was purchased from Pharmacia Biotech (Sweden). In this experiment, the 100 base pair ladder DNA was mixed with loading buffer $(0.25 \%$ Bromophenol Blue in $40 \%$ glycerol) at 5:1 and then adjusted to $0.5 \mu \mathrm{g} / \mu \mathrm{l}$ with Milli-Q water. Sample volume injected was $1 \mu \mathrm{l}$.

The DNA silver staining kit was also purchased from Pharmacia Biotech. All silver staining procedures were finished within $1 \mathrm{~h}$ at room temperature.

The channel structure in which the slab gel was cast was constructed of two polished glass plates $(80 \mathrm{~mm}$ long $\times 110 \mathrm{~mm}$ wide $\times 3 \mathrm{~mm}$ thick) and two Lumirror spacers $(80 \mathrm{~mm}$ long $\times 50 \mathrm{~mm}$ wide $\times 350 \mu \mathrm{m}$ thick). Components of the discontinuous slab gel were as follows: polyacrylamide $(3 \% \mathrm{~T}, 3 \% \mathrm{C})$ with $0.1 \mathrm{M} \mathrm{pH} 6.8$ Tris- $\mathrm{HCl}$ buffer was used as concentrating gel, and polyacrylamide $(7.5 \% \mathrm{~T}, 3 \% \mathrm{C})$ with $0.15 \mathrm{M} \mathrm{pH} 8.8$ Tris- $\mathrm{HCl}$ buffer was used as resolving gel. The cathodic buffer reservoir was filled with $0.025 \mathrm{M} \mathrm{pH} 8.3$ Tris-Glycine buffer solution and the anodic buffer reservoir with $0.15 \mathrm{M} \mathrm{pH} 8.8$ Tris- $\mathrm{HCl}$ buffer solution was used as running buffer. The concentrating gel was $10 \mathrm{~mm}$ in length and the resolving gel was $60 \mathrm{~mm}$ in length. For the conventional continuous slab gel experiment, a polyacrylamide gel $(7.5 \% \mathrm{~T}, 3 \% \mathrm{C}$ and was 200 $\mathrm{mm}$ long $\times 120 \mathrm{~mm}$ wide $\times 350 \mu \mathrm{m}$ thick) with $0.1 \mathrm{M} \mathrm{pH}$ 8.5 Tris-borate buffer containing $1 \mathrm{mM}$ EDTA was used. All gel solutions were diluted from 30\% T, 3\%C polyacrylamide stock solution and degassed under vacuum. Polymerization was initiated with $5 \mu \mathrm{l}$ of $10 \%$ $N, N, N^{\prime}, N^{\prime}$-tetramethylethylenediamine (TEMED) and 5 $\mu \mathrm{l}$ of $10 \%$ ammonium persulfate solution for $1 \mathrm{ml}$ gel solution. All procedures were carried out at room temperature.

\section{Safety concerns}

Care was taken when handling acrylamide monomers, as they are toxic.

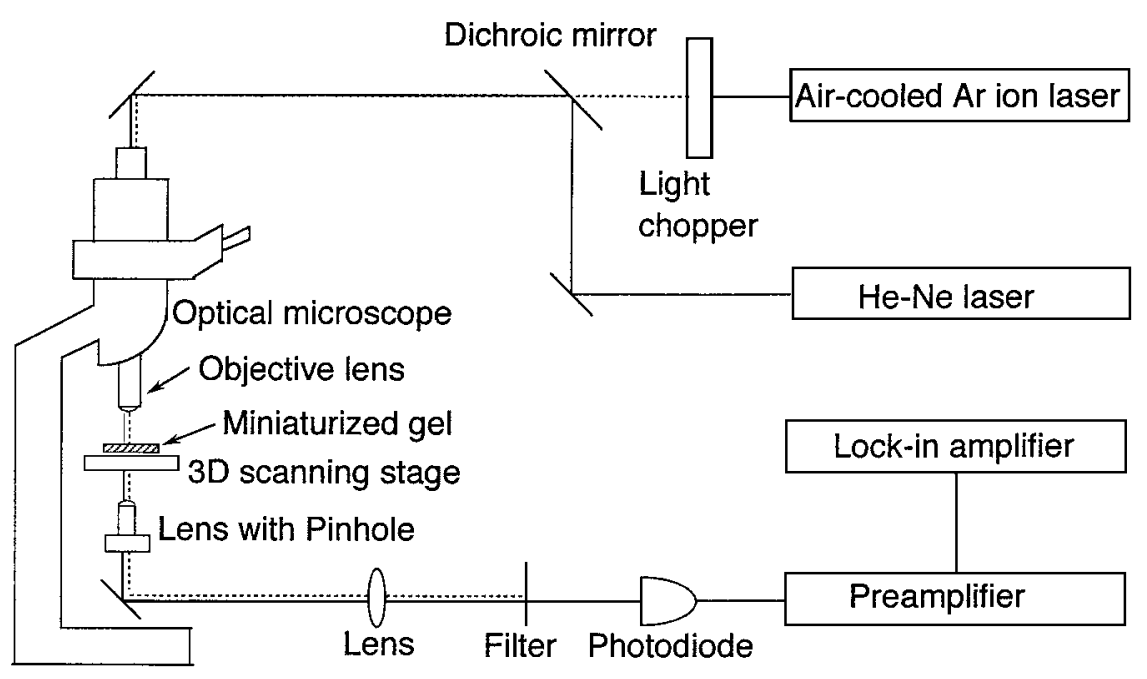

Fig. 1 Schematic illustration of thermal lens microscope (TLM) detection. 


\section{Results and Discussion}

\section{How to shorten the separation time}

In order to understand how to shorten the separation time, first we review what affects the separation time. The separation time $t$ and resolution $R_{\mathrm{S}}$ can be expressed by the following two equations: ${ }^{15}$

$$
\begin{aligned}
& t=X / m E \\
& R_{\mathrm{s}}=1.18 \Delta X / W_{1 / 2}
\end{aligned}
$$

in which $m$ is the ion mobility, $E$ is the field applied, $X$ is the migration distance, $\Delta X$ is the distance between the peak center of neighboring bands, and $W_{1 / 2}$ is the peak width at half of its maximum height.

From these two equations, we can see that the separation speed can be improved in two ways without resolution loss: 1) to use a higher field $E$ to get a shorter separation time $t$, and 2) to decrease $W_{1 / 2}$ so that a small $\Delta X$ will give the same $R_{\mathrm{s}}$. Assuming that $\Delta X$ is in proportion to $X$, we can shorten the migration distance and thus the separation time.

The first method is employed in ultrathin slab gel electrophoresis, capillary electrophoresis and microchip electrophoresis. In these cases, Joule heat on applying the voltage is dissipated efficiently. Therefore, bandbroadening from the temperature gradient can be minimized. An electric field as high as $100-300 \mathrm{~V} / \mathrm{cm}$ can be used, without causing a serious temperature gradient from Joule heat. Therefore, the resolution is comparable to that obtained in a moderate field because bandwidth remains almost unchanged, while fast separation can be obtained because of the high field applied. However, the electric field cannot be increased infinitely, because orientation of longer fragments will take place in higher fields and this will result in decreased resolution.

On the other hand, advantages of carrying out electrophoretic separation in a short slab gel have not been utilized so far. These advantages include the need for only small quantities of reagents and a small electrical supply, and ease in constructing the separation system and conditioning the environment of the whole system etc. Therefore, we thought it reasonable to attempt fast separation through the minimization of band-broadening and application of a short slab gel. That left another critical problem: how to sharpen the bands to improve the resolution.

As indicated by Luckey et al. ${ }^{16}$, the width of the DNA bands is determined by four factors: diffusion of sample injected, longitudinal diffusion during electrophoresis, thermal gradients from Joule heat, and spatial resolution of detector. It is easy to understand that if the migration distance is shortened, the longitudinal diffusion will decrease. And with the short migration distance, it is unnecessary to use a high field to obtain fast separation. Therefore, a moderate field is used and this will not result in a larger temperature gradient than in a conventional slab gel. As to band-broadening from diffusion of sample injected, we think that it can be reduced by using a discontinuous buffer system as described by Ornstein ${ }^{17}$, Chrambach and Jovin ${ }^{18}$, and Martin and Everaerts. ${ }^{19}$ In this experiment, three kinds of different buffers with a common counter ion were used as running buffer, concentrating buffer and resolving buffer, respectively. On application of electric field, a moving boundary was formed, causing the sample to be concentrated in the concentrating gel ${ }^{18}$, which is also known as stacking gel and is widely used in protein separation. The concentrated sample migrated into the resolving gel with reduced vertical spacing and was then separated. The last one, band-broadening from the detection method, seems to be the most difficult to overcome. Currently used detectors, such as UV-visible absorbance or LIF detectors, usually have a spatial resolution of $c a .50 \mu \mathrm{m}$, and this will yield a constant addition to the half-width of all the bands on the order of $c a .60 \mu \mathrm{m}^{16}$ (assuming that the excitation beam profiles are Gaussian). This is negligible when the migration distance is longer than $10 \mathrm{~cm}$ and the bandwidth is ca. $1 \mathrm{~mm}$. However, it cannot be neglected when the migration distance is shortened to several $\mathrm{cm}$ and the band is sharpened to $c a .100 \mu \mathrm{m}$. Therefore, a high spatial resolution detector is needed to detect the sharpened bands.

The spatial resolution of UV-visible absorbance or LIF detection can be improved by using a microscope to focus the excitation laser beam. However, the sensitivity of UV-visible absorbance is not high enough to be applied for detecting samples as thin as slab gels. The spatial resolution of LIF is usually affected by light scattering from the gel matrices which have strong light-scattering properties, and the random bursting of fluorescence. And a confocal LIF detector may be one choice to obtain high spatial resolution.

On the other hand, photothermal spectroscopy measures the changes in thermodynamic parameters of the sample caused by light absorption. Sample heating is a direct consequence of optical absorption, and photothermal spectroscopy signals are directly dependent on light absorption. Scattering and reflection losses do not produce photothermal signals. ${ }^{20}$ In the TLM instrument developed $^{21}$, the optical spatial resolution is determined by the spot size (typically $1 \mu \mathrm{m}$ ) of the excitation beam irradiated into the sample. TLM has been applied to other miniaturized systems and demonstrated to be a suitable detector for them. ${ }^{22-24}$ Then we used TLM to detect the DNA fragments separated in the slab gel with the intent of reducing band-broadening from the detector itself.

\section{Band sharpening effect of discontinuous buffer system}

Separation patterns of the miniaturized discontinuous slab gel and conventional continuous slab gel are compared in Fig. 2, and the separation conditions are summarized in Table 1. For conventional continuous slab 
a)

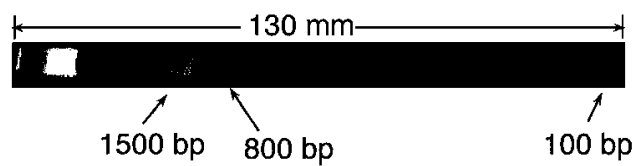

b)
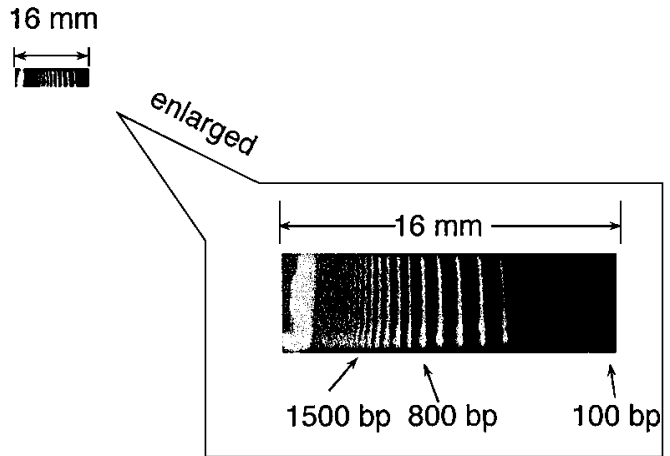

Fig. 2 Photographs of separated DNA samples (100 base pair ladder). a) Conventional gel; b) miniaturized gel.

Table 1 Comparison of miniaturized gel and conventional gel in separating 100 base pair ladder DNA fragments

\begin{tabular}{lcc}
\hline & Miniaturized gel & Conventional gel \\
\hline Separation time $(\mathrm{min})$ & 15 & 150 \\
Migration distance $(\mathrm{mm})$ & 18 & 130 \\
Electronic field $(\mathrm{V} / \mathrm{cm})$ & 30 & 18 \\
Sample volume $(\mu \mathrm{l})$ & 1 & 2 \\
Detected bands & 24 & 20 \\
\hline
\end{tabular}

gel electrophoresis, $150 \mathrm{~min}$ were required to obtain sufficient separation of the 100 base pair ladder DNA sample with an electric field of $18 \mathrm{~V} / \mathrm{cm}$ followed by silver staining detection. But using a miniaturized discontinuous slab gel, separation was finished in $15 \mathrm{~min}$ with an electric field of $30 \mathrm{~V} / \mathrm{cm}$ (as shown in Fig. 2b). Bandwidth in the miniaturized discontinuous slab gel was determined to be $100-350 \mu \mathrm{m}$, corresponding to $500-2000 \mu \mathrm{m}$ in the conventional slab gel.

Theoretically, we can predict that even though a DNA sample of very low concentration is injected, it would be concentrated to have a concentration of $[\mathrm{DNA}]^{19}$ by the concentrating gel described above.

$$
[\mathrm{DNA}]=\frac{m_{\mathrm{DAN}} Z_{\mathrm{Cl}}\left(m_{\mathrm{Cl}-}-m_{\text {Tris }}\right)}{m_{\mathrm{Cl}-} Z_{\mathrm{DNA}}\left(m_{\mathrm{DNA}}-m_{\mathrm{Tris}^{+}}\right)}\left[\mathrm{Cl}^{-}\right]
$$

where [DNA] is the concentration of concentrated DNA sample and $\left[\mathrm{Cl}^{-}\right]$is the concentration of $\mathrm{Cl}^{-}$in the concentrating gel; $Z$ is the ion charge and $m$ is the ion mobility. As a result, DNA was concentrated, accompanied by a decrease in the band width $\left(W_{1 / 2}\right)$ and an increase in $R_{\mathrm{s}}$.

Using Eq. (3), we calculated that DNA sample would be stacked into a thin layer of about $10 \mu \mathrm{m}$ in the concentrating gel. However, on entering the resolving gel,

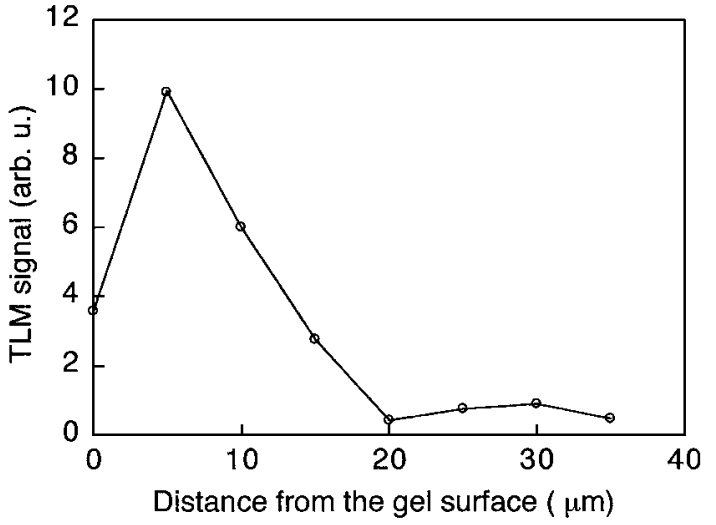

Fig. 3 TLM signal dependence on the focus point of the excitation beam.

DNA sample migrated slower than the anions of the cathodic buffer, and was separated in the same way as the continuous slab gel. Longitudinal diffusion occurred and a temperature gradient was generated, and these resulted in band-broadening. Therefore, the experimentally detected bandwidth was larger than that calculated by using Eq. (3).

\section{Detection of DNA bands using TLM}

TLM signals are affected by many factors, such as excitation beam power, frequency of the modulating chopper and focal position of the laser in the slab gel. ${ }^{21}$ In this experiment, we found that the focal point of the laser greatly affected the TLM signal and the $S / N$ ratio in the detection of silver stained slab gel. Therefore, we investigated changes of the TLM signal with the focal position of the laser in the silver stained gel to obtain the best detection conditions. As shown in Fig. 3 , the largest signal could be obtained when the probe laser was focused onto a point $5 \mu \mathrm{m}$ beneath the gel surface. We attributed this to a characteristic of silver staining, that it takes place more easily near the surface. Also we found that the best $S / N$ ratio could be obtained if the focal point was set at $5 \mu \mathrm{m}$ beneath the gel surface. Because the TLM signal changed greatly with the focal position, it was also necessary to adjust the horizontal level of the gel to keep the focus at the same depth in the gel during horizontal scanning.

DNA bands in the miniaturized discontinuous slab gel that could not be discriminated visually in Fig. $2 b$ were successfully detected using TLM by scanning the gel. These results are shown in Fig. 4. DNA bands of more than $2000 \mathrm{bp}$ were successfully detected. The results were better than those obtained by a conventional continuous slab gel and the separation time was only one-tenth. As shown in Fig. 4, the DNA band width was sharpened to about $100 \mu \mathrm{m}$. We thought that this should be attributed to the application of a discontinuous buffer system and a high spatial resolution detector. Therefore, better resolution was obtained in the miniaturized discontinuous slab gel. 


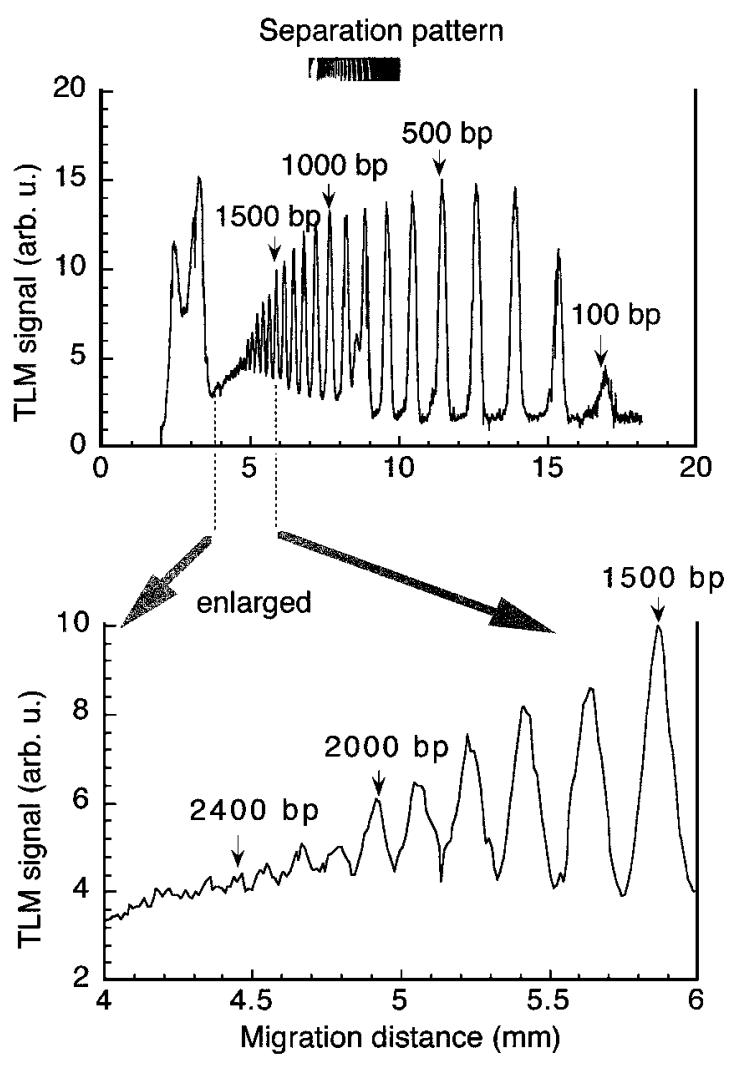

Fig. 4 High spatial resolution detection of DNA fragments by TLM. Excitation beam: air-cooled Ar ion laser, $488 \mathrm{~nm}, 6$ $\mathrm{mW}$; probe beam: He-Ne laser, $633 \mathrm{~nm}, 1 \mathrm{~mW}$. Frequency of light chopper: $1173 \mathrm{~Hz}$. Time constant: $1.25 \mathrm{~s}$. Interval of 3D scanning stage: $10 \mu \mathrm{m} / \mathrm{step}$.

We showed that fast separation in slab gel electrophoresis could be obtained with a short migration distance and minimized band-broadening. These results indicated that fast separation could be obtained through miniaturization of the slab gel. We could miniaturize the slab gel not only in length, but also in thickness, because a thinner slab gel would allow a higher field to be used to further shorten the separation time. It has been demonstrated that the resolution or the number of theoretical plates $N$ of a given gel is related to the applied voltage as follows: ${ }^{25}$

$$
N=\frac{\mu_{\mathrm{e}} V}{2 D}
$$

where $V$ is the applied voltage and $D$ is the diffusion coefficient. Other factors being held constant, increasing the voltage will also increase the number of theoretical plates and hence the system efficiency. Considering the high spatial resolution of TLM, which is about $c a .1 \mu \mathrm{m}$, we could use an ultra-thin slab gel as thin as $c a .10 \mu \mathrm{m}$, if necessary. Our own experiences have shown that it is not too difficult to cast and operate a miniaturized slab gel as thin as $c a .10 \mu \mathrm{m}$. Combining with the inherent properties of parallel sep- arations in slab gel, we could obtain high throughput separation in a miniaturized slab gel. Therefore, we predict that miniaturized slab gel electrophoresis, together with high spatial resolution detection of TLM, will become a useful tool for high throughput separation of DNA, such as DNA sequencing, genetic diagnosis and forensic identification analyses.

\section{References}

1. F. Sanger, S. Nicklen and A. R. Coulson, Proc. Natl. Acad. Sci. U.S.A., 74, 5463 (1977).

2. A. M. Maxam and W. Gilbert, Proc. Natl. Acad. Sci. U.S.A., 74, 560 (1977).

3. G. P. S. Raghava, Biotechniques, 17, 100 (1994).

4. C. L. Smith and C. R. Cantor, TIBS, 12, 284 (1987).

5. R. A. Kroczek, J. Chromatog.: Biomed. Appl., 618, 133 (1993).

6. A. Schmitz and D. J. Galas, Nucleic Acids Res., 6, 111 (1979).

7. P. B. Hietpas, K. M. Bullard, D. A. Gutman and A. G. Ewing, Anal. Chem., 69, 2292 (1997).

8. J. A. Luckey, H. Drossman, A. J. Kostichka, D. A. D'Cunha, T. B. Norris and L. M. Smith, Nucleic Acid Res., 18, 4417 (1990).

9. P. Carninci, F. Volpatti and C. Schneider, Electrophoresis, 16, 1836 (1995).

10. Y. Baba, R. Tomisaki, C. Sumita, I. Morimoto, S. Sugita, M. Tsuhako, T. Miki and T. Ogihara, Electrophoresis, 16, 1437 (1995).

11. J. Y. Yan, N. Best, J. Z. Zhang, H. J. Ren, R. Jiang, J. Hou and N. J. Dovichi, Electrophoresis, 17, 1037 (1996).

12. X. C. Huang, M. A. Quesada and R. A. Mathies, Anal. Chem., 64, 2149 (1992).

13. A. T. Woolley, G. F. Sensabaugh and R. M. Mathies, Anal. Chem., 69, 2181 (1997).

14. D. Schmaizing, A. Adourian, L. Koutny, L. Ziaugra, P. Matsudaira and D. Ehrlich, Anal. Chem., 70, 2303 (1998).

15. J. J. Kirkland, W. W. Yau, H. J. Stoklosa and C. H. J. Dilks, J. Chromatogr. Sci., 15, 303 (1977).

16. J. A. Luckey, T. B. Norris and L. M. Smith, J. Phys. Chem., 97, 3067 (1993).

17. L. L. Ornstein, Ann. N. Y. Acad. Sci., 121, 321 (1964).

18. A. Chrambach and T. M. Jovin, Electrophoresis, 4, 190 (1983).

19. A. J. P. Martin and F. M. Everaerts, Proc. Roy. Soc. London A, 316, 493 (1970).

20. S. E. Bialkowski, "Photothermal Spectroscopy Methods for Chemical Analysis", John Wiley and Sons, New York, Chichester, Brisbane, Toronto, Singapore, 1996.

21. M. Harada, M. Shibata, T. Kitamori and T. Sawada, Anal. Chim. Acta, 299, 343 (1995).

22. H. Kimura, M. Mukaida, T. Kitamori and T. Sawada, Anal. Sci., 13, 729 (1997).

23. M. Harada, K. Iwamoto, T. Kitamori and T. Sawada, Anal. Chem., 65, 2938 (1993).

24. M. Harada, M. Shibata, T. Kitamori and T. Sawada, Anal. Sci., submitted.

25. J. C. Giddings, Separation Science, 4, 181 (1969).

(Received September 28, 1998) (Accepted January 13, 1999) 